\title{
Heavy flavour spectroscopy and hadron properties from LHCb
}

\author{
Antimo Palano ${ }^{1, *}$ \\ ${ }^{1}$ INFN and University of Bari, Via Orabona 4, 70125 Bari, Italy
}

\begin{abstract}
The LHCb experiment is designed to study the properties and decays of heavy flavored hadrons produced in pp collisions at the LHC. The data collected enables precision spectroscopy studies of beauty and charm hadrons. The latest results on spectroscopy of conventional and exotic hadrons are reviewed.
\end{abstract}

\section{Introduction}

In the simple quark model, only two types of quark combinations are required to account for the existing hadrons, i.e. $q \bar{q}$ combinations form mesons, while baryons are made up of three quarks. However, in the quark model proposed by Gell-Mann and Zweig in 1960s [1] other SU(3) color-neutral combinations of quarks and gluons such as $g g$ glueballs, $q \bar{q} g$ hybrids, $q \bar{q} q \bar{q}$ tetraquarks, $q \bar{q} q q q$ pentaquarks etc. are allowed. The world's largest data sample of beauty and charm hadrons collected by LHCb provides great opportunities for studying the production and properties of heavy hadrons. The present document describes some recent results from $\mathrm{LHCb}$ in exotic and standard hadron spectroscopy.

\section{The observation of pentaquarks in $\Lambda_{b}^{0} \rightarrow J / \psi p K^{-}$}

Evidences/observations of pentaquarks candidates were reported around 2005 by several experiments, always with low statistics and approximate statistical treatment of the data. For a summary and references see ref. [2]. High statistics searches gave negative results (see for example ref. [3]).

LHCb experiment studied the decay of $\Lambda_{b}^{0} \rightarrow J / \psi p K^{-}$[4]. A sample of 26,007 \pm 166 signal candidates, on $5.4 \%$ background, within $\pm 15 \mathrm{MeV}$ of the $J / \psi p K^{-}$mass peak has been selected using the full Run I dataset. An anomalous peaking structure in the $J / \psi p$ invariant mass spectrum was observed on the Dalitz plot. This structure can also be seen in the invariant mass projections shown in Fig. 1. If the peak structure observed in Fig. 1(b) represents a resonance strongly decaying into $J / \psi p$, the minimal valence quarks should be $c \bar{c} u u d$, a charmonium pentaquark state. A full six-dimensional amplitude fit with resonance invariant mass, three helicity angles and two differences between decay planes has been applied to describe the data. It allowed interference between the decays $\Lambda_{b}^{0} \rightarrow J / \psi \Lambda^{*}$ and $\Lambda_{b}^{0} \rightarrow P_{c}^{+} K^{-}$, with $\Lambda^{*} \rightarrow p K^{-}$and $P_{c}^{+} \rightarrow J / \psi p$, where $P_{c}^{+}$indicates the pentaquark candidate. The amplitude model in the fit contains 14 well-defined $\Lambda^{*}$ states listed by Particle Data Group [5] and two $P_{c}^{+}$states: $P_{c}(4380)^{+}$and $P_{c}(4450)^{+}$. With a full amplitude analysis, the masses of these two states are measured to be $4380 \pm 8$ (stat) \pm 29 (syst) $\mathrm{MeV}$ and $4449.8 \pm 1.7$ (stat) \pm 2.5 (syst) $\mathrm{MeV}$, with the widths

\footnotetext{
*e-mail: antimo.palano@ba.infn.it
} 

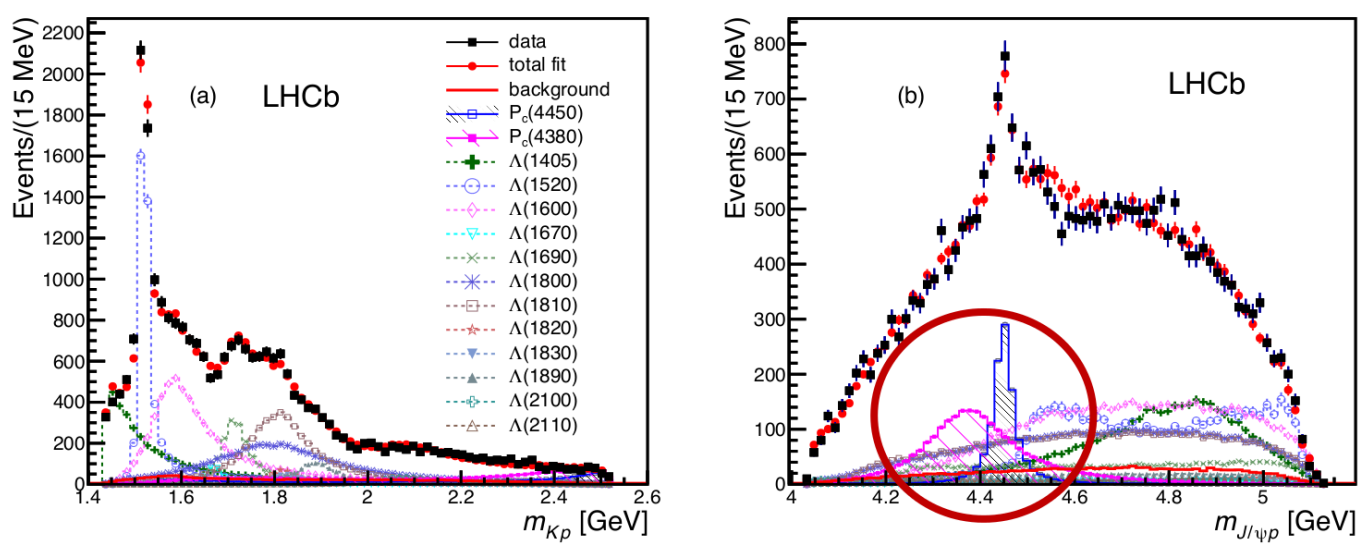

Figure 1. Dalitz plot projections of $\Lambda_{b}^{0} \rightarrow J / \psi p K^{-}$with superimposed the results from the amplitude analysis.

$205 \pm 18$ (stat) \pm 86 (syst) $\mathrm{MeV}$ and $39 \pm 5$ (stat) \pm 19 (syst) $\mathrm{MeV}$, respectively. The preferred spin-parity values are $\left(3 / 2^{-}, 5 / 2^{+}\right),\left(3 / 2^{+}, 5 / 2^{-}\right)$and $\left(5 / 2^{+}, 3 / 2^{-}\right)$, where the first one is the $J^{P}$ assignment given by the best fit. This analysis has been extended with a model-independent approach [6] which gave consistent results. Results consistent with expectations have also been obtained from the amplitude analysis of the Cabibbo-suppressed decay $\Lambda_{b}^{0} \rightarrow J / \psi p \pi^{-}$[7].

\section{Confirmation of resonant nature of $Z_{c}(4430)^{-}$}

The $Z_{c}(4430)^{-}$state was first reported by the Belle collaboration [8] as a charged resonance structure in the $\psi^{\prime} \pi^{-}$invariant mass distribution in the decay $B^{0} \rightarrow \psi^{\prime} K^{+} \pi^{-}$. The BaBar collaboration could explain the enhancement as a reflection of the known $K^{*}$ states [9].

The LHCb experiment collected about 25,000 $B^{0}$ signal events, a yield one order of magnitude larger than the BaBar or Belle datasets, and observed the $Z_{c}(4430)^{-}$with a significance larger than $13.9 \sigma[10]$. The LHCb collaboration performed both a model independent analysis as well as a full amplitude analysis. The projection of the fit including the $Z_{c}(4430)^{-}$state is shown in Fig. 2 and evidences a good description of the data. The measured parameters are $M\left(Z_{c}\right)=4475 \pm 7_{-25}^{+15}$ $\mathrm{MeV}, \Gamma\left(Z_{c}\right)=172 \pm 13_{34}^{+37} \mathrm{MeV}$. The Argand diagram of the $Z_{c}(4430)^{-}$amplitude (Fig. 2) shows the resonance behavior for the first time. The spin-parity was measured to be $1^{+}$, by excluding other hypotheses by at least $9.7 \sigma$. For a charged charmonium state, $Z_{c}(4430)^{-}$has a minimum quark content of $c \bar{c} u \bar{d}$ which clearly does not fit into the traditional quark model.

\section{Study of $B \rightarrow J / \psi \phi K$}

There has been a great deal of experimental and theoretical interest in $J / \psi \phi$ mass structures in $B^{+} \rightarrow J / \psi \phi K^{+}$decays since the CDF collaboration presented $3.8 \sigma$ evidence for a near-threshold $X(4140)$ mass peak, with width $\Gamma=11.7 \mathrm{MeV}$ [11]. Subsequent measurements resulted in a confusing experimental situation. LHCb experiment has reconstructed $4289 \pm 151 \mathrm{~B}^{+} \rightarrow J / \psi \phi \mathrm{K}^{+}$decays, with roughly uniform efficiency and a relatively low background across the entire $J / \psi \phi$ mass range [12]. A full amplitude analysis has been performed, including resonant contributions from $K^{*}$ resonances 

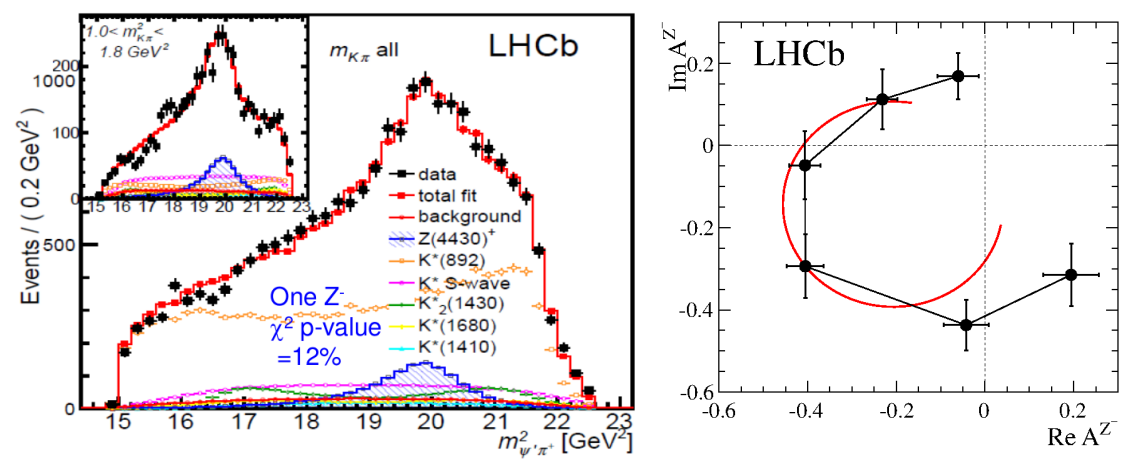

Figure 2. (Left) $\psi^{\prime} \pi^{-}$mass distribution from $\bar{B}^{0} \rightarrow \psi^{\prime} \pi^{-} K^{+}$with superimposed the results from the amplitude analysis. (Right) Argand diagram for $Z_{c}(4430)^{-}$.

Table 1. Parameters of the resonances decaying to $J / \psi \phi$ resulting from the amplitude analysis of $B^{+} \rightarrow J / \psi \phi K^{+}$

\begin{tabular}{lcccc}
\hline Resonance & $\sigma$ & $J^{P C}$ & $\mathrm{M}(\mathrm{MeV})$ & $\Gamma(\mathrm{MeV})$ \\
\hline $\mathrm{X}(4140)$ & 8.4 & $1^{++}$ & $4160 \pm 4_{-3}^{+5}$ & $83 \pm 21_{-14}^{+21}$ \\
$\mathrm{X}(4274)$ & 5.8 & $1^{++}$ & $4273 \pm 8_{-4}^{+17}$ & $56 \pm 11_{-11}^{+8}$ \\
$\mathrm{X}(4500)$ & 6.1 & $0^{++}$ & $4506 \pm 11_{-15}^{+12}$ & $92 \pm 21_{-20}^{+21}$ \\
$\mathrm{X}(4700)$ & 5.6 & $0^{++}$ & $4704 \pm 10_{-24}^{+14}$ & $120 \pm 31_{-33}^{+42}$ \\
\hline
\end{tabular}

decaying to $\phi K^{+}$. Four resonant contributions in the $J / \psi \phi$ final state are found to contribute, whose parameters and quantum numbers are given in Table 1 . The fit results are superimposed to the $J / \psi \phi$ mass spectrum in fig. 3 . It can be noted that the $X(4140)$ width is substantially larger than previously
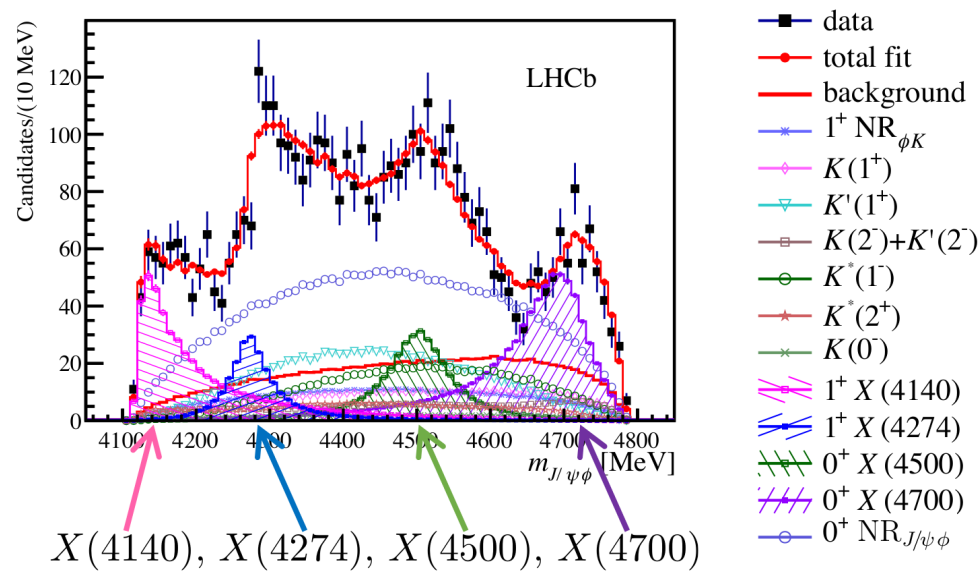

Figure 3. $J / \psi \phi$ mass projection with superimposed the fit result from the amplitude analysis of $B^{+} \rightarrow J / \psi \phi K^{+}$

determined. The interpretation of these states is still an open issue. 


\section{Observation of five new $\Omega_{c}^{0}$ states}

The spectroscopy of singly charmed baryons $c q q^{\prime}$ is intricate. With three quarks and numerous degrees of freedom, many states are expected. Among the expected charmed baryon states the spectrum of $\Omega_{c}$ baryons, which have quark content $c s s$ and isospin zero, was largely unknown: only the $\Omega_{c}$ and $\Omega_{c}(2770)^{0}$, presumed to be the $J^{P}=1 / 2^{+}$and $3 / 2^{+}$ground states, were observed.

The LHCb experiment has reconstructed a large $\left(\approx 10^{6}\right.$ events $)$ and high-purity $(83 \%)$ sample of $\Xi_{c}^{+}$baryons, reconstructed in the Cabibbo-suppressed decay mode $p K^{+} \pi^{-}$[13]. Surprisingly, in the $\Xi_{c}^{+} K^{-}$mass spectrum, shown in Fig. 4, five new, narrow excited $\Omega_{c}$ states are observed: the $\Omega_{c}(3000)^{0}, \Omega_{c}(3050)^{0}, \Omega_{c}(3066)^{0}, \Omega_{c}(3090)^{0}$, and $\Omega_{c}(3119)^{0}$. The measured masses and widths are reported in Table 2. The data indicate also the presence of a broad structure around $3188 \mathrm{MeV}$ that is fitted as a single resonance but could be produced in other ways, for example as a superposition of several states. In addition, the partially reconstructed decay $\Omega_{c}(3066)^{0}, \Omega_{c}(3090)^{0}$ and $\Omega_{c}(3119)^{0}$ in their possible decay to $\Xi_{c}^{\prime+} K^{-}$are observed via their feed-down in the low mass region. This observation has generated large interest, especially for the presence of very narrow states, and many phenomenological models have been proposed, including the possibility of the presence of pentaquark states.

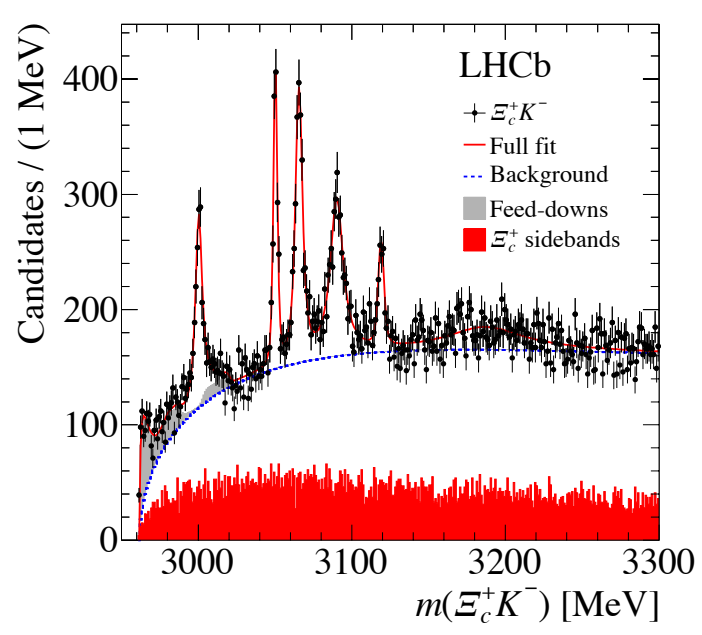

Figure 4. $\Xi_{c}^{+} K^{-}$mass spectrum with the observation of five new $\Omega_{c}$ states.

\section{Observation of the double charmed baryon $\Xi_{c c}^{++}$}

The constituent quark model of hadrons predicts the existence of three weakly decaying ground state $J^{P}=1 / 2^{+}$baryons with two charmed valence quarks: $\Xi_{c c}^{+}(\mathrm{ccd})$ and $\Xi_{c c}^{++}(\mathrm{ccu})$, which form an isospin doublet, and $\Omega_{c c}^{+}$(ccs). The only previously published evidence of their existence comes from the observations of the $\Xi_{c c}^{+}$baryon claimed by the SELEX collaboration [14]. They observed narrow peaking low statistics structures at a mean mass of $3518.7 \pm 1.7 \mathrm{MeV}$ in the mass spectra of $\Lambda_{c}^{+} K^{-} \pi^{+}$ and $p D^{+} K^{-}$.

LHCb experiment has searched for double charmed baryon states using the decay mode $\Xi_{c c}^{++} \rightarrow$ $\Lambda_{c} K^{-} \pi^{+} \pi^{+}$, with $\Lambda_{c} \rightarrow p K^{-} \pi^{+}$(which has a $10 \%$ branching fraction) [15]. The $\Lambda_{c} K^{-} \pi^{+} \pi^{+}$mass 
Table 2. Measured parameters of the five new $\Omega_{c}$ states.

\begin{tabular}{lcc}
\hline Resonance & Mass $(\mathrm{MeV})$ & $\Gamma(\mathrm{MeV})$ \\
\hline$\Omega_{c}(3000)^{0}$ & $3000.4 \pm 0.2 \pm 0.1_{-0.5}^{+0.3}$ & $4.5 \pm 0.6 \pm 0.3$ \\
$\Omega_{c}(3050)^{0}$ & $3050.2 \pm 0.1 \pm 0.1_{-0.5}^{+0.3}$ & $0.8 \pm 0.2 \pm 0.1$ \\
& & $<1.2 \mathrm{MeV}, 95 \% \mathrm{CL}$ \\
$\Omega_{c}(3066)^{0}$ & $3065.6 \pm 0.1 \pm 0.3_{-0.5}^{+0.3}$ & $3.5 \pm 0.4 \pm 0.2$ \\
$\Omega_{c}(3090)^{0}$ & $3090.2 \pm 0.3 \pm 0.5_{-0.5}^{+0.3}$ & $8.7 \pm 1.0 \pm 0.8$ \\
$\Omega_{c}(3119)^{0}$ & $3119.1 \pm 0.3 \pm 0.9_{-0.5}^{+0.3}$ & $1.1 \pm 0.8 \pm 0.4$ \\
& & $<2.6 \mathrm{MeV}, 95 \% \mathrm{CL}$ \\
\hline$\Omega_{c}(3188)^{0}$ & $3188.1 \pm 4.8 \pm 12.7$ & $60 \pm 15 \pm 11$ \\
\hline
\end{tabular}

spectrum is shown in Fig. 5(a) where a prominent signal can be observed. LHCb observes $313 \pm 33$
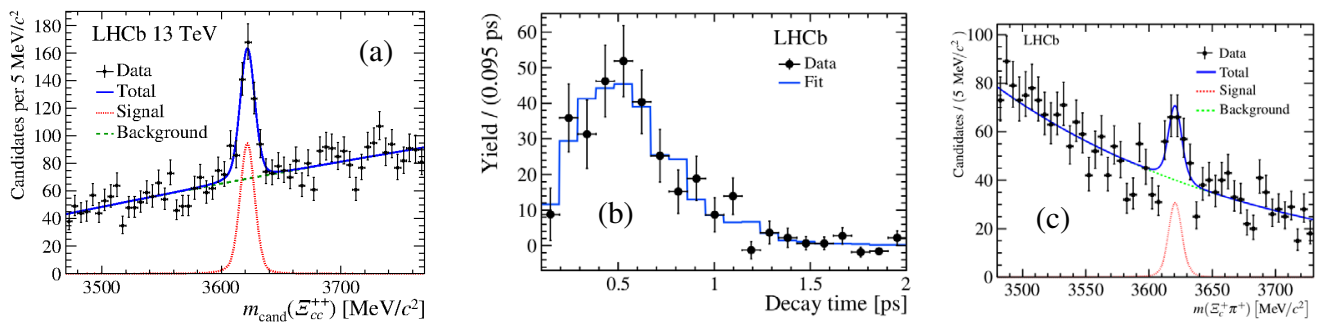

Figure 5. (a) $\Lambda_{c} K^{-} \pi^{+} \pi^{+}$mass spectrum with the observation of $\Xi_{c c}^{++}$. (b) Background-subtracted decay-time distribution of selected $\Xi_{c c}^{++}$candidates with superimposed fit results. (c) Observation of $\Xi_{c c}^{++} \rightarrow \Xi_{c}^{+} \pi^{+}$.

decays with a significance of $>12 \sigma$ of a $\Xi_{c c}^{++}$state that has a mass of $m\left(\Xi_{c c}^{++}\right)=3621.40 \pm 0.72($ stat $) \pm$ 0.27 (syst) $\pm 0.14\left(\Lambda_{\mathrm{c}}\right) \mathrm{MeV}$ and a width that is much smaller than experimental resolution and that is consistent with a particle that flies a significant distance before decaying. This is the first published observation of the $\Xi_{c c}^{++}$baryon. The published SELEX $\Xi_{c c}^{+}$state has a mass that is $103 \pm 2 \mathrm{MeV}$ smaller than the $\Xi_{c c}^{++}$state, so the two observations are inconsistent with being isospin partners. A consistent interpretation of the two observations may prove challenging.

\section{Lifetime measurement of $\Xi_{c c}^{++}$}

LHCb experiment has performed a $\Xi_{c c}^{++}$lifetime measurement relative to that of the $\Lambda_{b}^{0} \rightarrow \Lambda_{c}^{+} \pi^{-} \pi^{+} \pi^{-}$ which has the same topology [16]. An unbinned maximum likelihood fit to the background subtracted (using the sFit method) $\Xi_{c c}^{++}$decay time distribution has been performed using the function

$$
R(t)=\frac{f_{\Xi_{c c}^{++}}}{f_{\Lambda_{b}^{0}}} \times \frac{\epsilon_{\Lambda_{b}^{0}}(t)}{\epsilon_{\Xi_{c c}^{++}}(t)}=R(0) \times e^{-\left(\frac{1}{\tau_{\Xi_{c c}^{++}}^{++}}-\frac{1}{\tau} \Lambda_{b}^{0}\right) t},
$$

where $\epsilon$ indicate the time dependent efficiencies. The fitted and background subtracted $\Xi_{c c}^{++}$lifetime distribution is shown in Fig. 5(b). The measured lifetime is

$$
\tau_{\Xi_{c c}^{++}}=0.256_{-0.022}^{+0.024} \pm 0.014 \mathrm{ps}
$$


in the lower end but compatible with the expected theoretical predictions. This confirms the weakly decay nature of the newly discovered $\Xi_{c c}^{++}$state.

\section{Observation of a new $\Xi_{c c}^{++} \rightarrow \Xi_{c}^{+} \pi^{+}$decay mode}

Searching for new decay modes is critical to understand the dynamics of weak decays of doubly heavy baryons. The present $\mathrm{LHCb}$ analysis makes use of a data sample of $1.7 \mathrm{fb}^{-1}$ collected at $13 \mathrm{TeV}$ [17]. The normalization channel $\Xi_{c c}^{++} \rightarrow \Lambda_{c} K^{-} \pi^{+} \pi^{+}$is used and a significant peak (5.9 $\left.\sigma\right)$ of $91 \pm 20$ events in the $\Xi_{c}^{+} \pi^{+}$mass spectrum (shown in Fig. 5(c)), where $\Xi_{c}^{+} \rightarrow p K^{-} \pi^{+}$is observed. The resulting mass measurement is $m\left(\Xi_{c c}^{++}\right)=3620 \pm 1.5$ (stat) \pm 0.4 (sys) $\pm 0.3\left(\Xi_{\mathrm{c}}^{+}\right) \mathrm{MeV} / \mathrm{c}^{2}$, consistent with the previous measurement. The ratio of branching fractions is also measured as

$$
\frac{\mathcal{B}\left(\Xi_{c c}^{++} \rightarrow \Xi_{c}^{+} \pi^{+}\right) \times \mathcal{B}\left(\Xi_{c}^{+} \rightarrow p K^{-} \pi^{+}\right)}{\mathcal{B}\left(\Xi_{c c}^{++} \rightarrow \Lambda_{c} K^{-} \pi^{+} \pi^{+}\right) \times \mathcal{B}\left(\Lambda_{c} \rightarrow p K^{-} \pi^{+}\right)}=0.035 \pm 0.009 \text { (stat) } \pm 0.003 \text { (syst) }
$$

\section{Measurement of the $\Omega_{c}$ baryon lifetime}

Charm baryon lifetimes are known much less precisely than charm meson ones. The expected lifetime hierarchy should be: $\tau_{\Xi_{c}^{+}}>\tau_{\Lambda_{c}^{+}}>\tau_{\Xi_{c}^{0}}>\tau_{\Omega_{c}^{0}}$ and current measurements are consistent with this. LHCb experiment makes use a sample of approximately $10^{3}$ semileptonic $\Omega_{b}^{-} \rightarrow \Omega_{c}^{0} \mu^{-} \bar{v}_{\mu} X$, with $\Omega_{c}^{0} \rightarrow p K^{-} K^{-} \pi^{+}[18]$ and a dataset of $3 \mathrm{fb}^{-1}$. To reduce uncertainties the ratio with respect to the $D^{+} \rightarrow K^{-} \pi^{+} \pi^{+}$lifetime is measured. The $\Omega_{c}^{0}$ signal is shown in Fig. 6 together with the decay time distribution. The fitted $\Omega_{c}^{0}$ lifetime is $\tau_{\Omega_{c}^{0}}=268 \pm 21_{\text {stat }} \pm 10_{\text {syst }} \pm 2 D_{D^{+}} f s$, four times larger than and
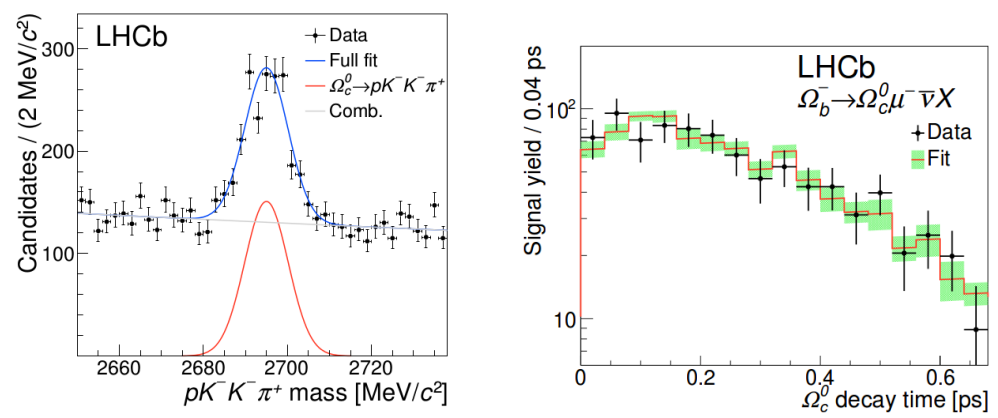

Figure 6. (Left) $\Omega_{c}^{0} \rightarrow p K^{-} K^{-} \pi^{+}$candidates and (Right) $\Omega_{c}^{0}$ decay time distribution with superimposed fit result.

inconsistent with the world average value of $(69 \pm 12)$ fs. The present measurement produces the modified lifetimes hierarchy as $\tau_{\Xi_{c}^{+}}>\tau_{\Omega_{c}^{0}}>\tau_{\Lambda_{c}^{+}}>\tau_{\Xi_{c}^{0}}$.

\section{Observation of a new $\Xi_{b}^{-}$resonance}

A new $\Xi_{b}(6227)^{-}$state is observed by LHCb decaying to both $\Xi_{b}^{0} \pi^{-}$and $\Lambda_{b}^{0} K^{-}$[19]. The analysis makes use of a dataset of $1.0 \mathrm{fb}^{-1}$ at $7 \mathrm{TeV}, 2.0 \mathrm{fb}^{-1}$ at $8 \mathrm{TeV}$ and $1.5 \mathrm{fb}^{-1}$ at $13 \mathrm{TeV}$. Three independent reconstructed decay chains are used: $\Xi_{b}(6227)^{-} \rightarrow \Lambda_{b}^{0}\left(\rightarrow \Lambda_{c}^{+} \pi^{-}\right) K^{-}, \Xi_{b}(6227)^{-} \rightarrow \Xi_{b}^{0}(\rightarrow$ $\left.\Xi_{c}^{+} \mu^{-} X\right) \pi^{-}$, and $\Xi_{b}(6227)^{-} \rightarrow \Lambda_{b}^{0}\left(\rightarrow \Lambda_{c}^{+} \mu^{-} X\right) K^{-}$. In the case of semileptonic decays the missing $p_{v}$ 

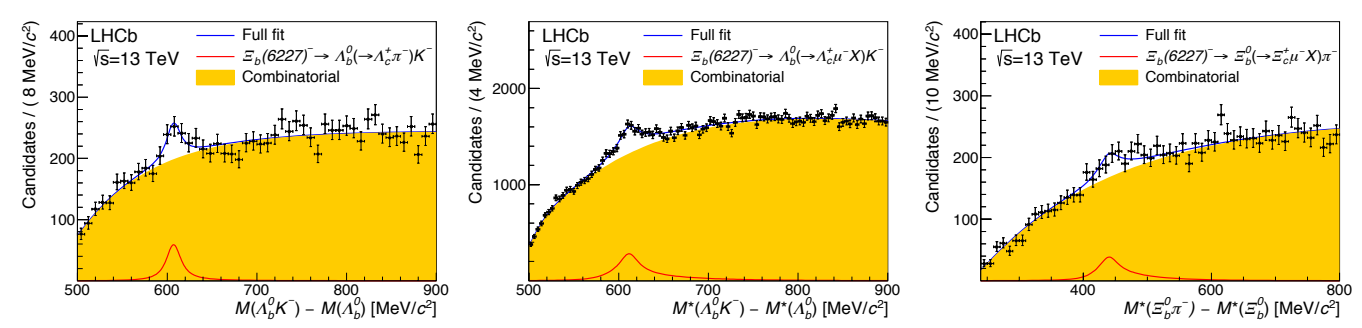

Figure 7. Observation of the new $\Xi_{b}(6227)^{-}$state in the three reconstructed decay modes for the $13 \mathrm{TeV}$ data.

momentum is reconstructed assuming a zero-mass particle that balances the momentum transverse to the direction of $\Lambda_{b}^{0} / \Xi_{b}^{0}$. The total invariant mass is then constrained to have the known $\Lambda_{b}^{0} / \Xi_{b}^{0}$ mass. The new resonance is observed in three different mass spectra as shown in Fig. 7 for the $13 \mathrm{TeV}$ data. Similar signals are observed in the $7 / 8 \mathrm{TeV}$ data. The new state could be identified as $\Xi_{b}(1 P)$ or $\Xi_{b}(2 S)$ states. Resonance resonance parameters are

$m_{\Xi_{b}(6227)^{-}}=6226.9 \pm 2.0$ stat \pm 0.3 syst $\pm 0.2\left(\Lambda_{b}^{0}\right) \mathrm{MeV} / c^{2}, \Gamma_{\Xi_{b}(6227)^{-}}=18.1 \pm 5.4$ stat $\pm 1.8 \mathrm{syst} \mathrm{MeV} / c^{2}$.

\section{References}

[1] M. Gell-Mann, Phys. Lett. 8, 214 (1964).

[2] A. Dzierba, C. Mayer and A. Szczepaniak, hep-ex/04120 (2005).

[3] B. Aubert et al. [BaBar Collaboration], Phys. Rev. Lett. 95, 042002 (2005). J. M. Link et al. [FOCUS Collaboration], Phys. Lett. B 639, 604 (2006).

[4] R. Aaij et al. [LHCb Collaboration], Phys. Rev. Lett. 115, 072001 (2015).

[5] C. Patrignani et al. (Particle Data Group), Chin. Phys. C, 40, 100001 (2016) and 2017 update.

[6] R. Aaij et al. [LHCb Collaboration], Phys. Rev. Lett. 117, no. 8, 082002 (2016).

[7] R. Aaij et al. [LHCb Collaboration], Phys. Rev. Lett. 117, no. 8, 082003 (2016).

[8] S. K. Choi et al. [Belle Collaboration], Phys. Rev. Lett. 100, 142001 (2008).

[9] B. Aubert et al. [BaBar Collaboration], Phys. Rev. D 79, 112001 (2009).

[10] R. Aaij et al. [LHCb Collaboration], Phys. Rev. Lett. 112, no. 22, 222002 (2014).

[11] T. Aaltonen et al., [CDF Collaboration], Phys. Rev. Lett. 102, 242002 (2009).

[12] R. Aaij et al. [LHCb Collaboration], Phys. Rev. Lett. 118, 022003 (2017).

[13] R. Aaij et al. [LHCb Collaboration], Phys. Rev. Lett. 118, 182001 (2017).

[14] A. Ocherashvili et al. [SELEX Collaboration], Phys. Lett. B 628, 18 (2005).

[15] R. Aaij et al. [LHCb Collaboration], Phys. Rev. Lett. 119, 112001 (2017).

[16] R. Aaij et al. [LHCb Collaboration], Phys. Rev. Lett. 121 (2018) no.5, 052002.

[17] R. Aaij et al. [LHCb Collaboration], arXiv:1807.01919 [hep-ex].

[18] R. Aaij et al. [LHCb Collaboration], Phys. Rev. Lett. 121 (2018) 092003.

[19] R. Aaij et al. [LHCb Collaboration], Phys. Rev. Lett. 121 (2018) no.7, 072002. 